J. Clin. Chem. Clin. Biochem.

Vol. 28, 1990, pp. $619-626$

(C) 1990 Walter de Gruyter \& Co. Berlin - New York

\title{
Plasma Carnitine Levels in Liver Cirrhosis: Relationship with Nutritional Status and Liver Damage
}

\author{
By P. Amodio, P. Angeli, C. Merkel, Francesca Menon and A. Gatta \\ Institute of Clinical Medicine, University of Padua, Italy
}

(Received September 6, 1989/May 3, 1990)

\begin{abstract}
Summary: The plasma level of carnitine, a co-factor involved in many metabolic reactions, is high in alcoholic liver cirrhosis, due to an increased amount of esterified carnitine. To determine if this alteration is linked to alcoholic liver disease or to liver cirrhosis per se, total carnitine, free carnitine, total esterified carnitine, short chain acylcarnitine and long chain acylcarnitine were measured in 41 patients suffering from liver cirrhosis of different aetiology and severity. In 19 of these patients, acetylcarnitine was also measured. Moreover, multivariate analysis was performed to assess the association of carnitine plasma levels with nutritional and liver disease indices.
\end{abstract}

Of the nutritional indices (creatinine/height ratio, mid upper arm muscle circumference and triceps skinfold) only triceps skinfold appeared to be weakly correlated with carnitine (with long chain acylcarnitine).

Significantly high levels of acetylcarnitine, short chain acylcarnitine, total esterified carnitine and total carnitine were found in cirrhotics independently of the aetiology of cirrhosis, even though a trend towards higher levels of acetylcarnitine was evident in heavy drinkers.

Direct correlations of $\gamma$-glutamyltransferase with acetylcarnitine, acetylcarnitine/free carnitine, short chain acylcarnitine/free carnitine and total esterified carnitine/free carnitine were found. Carnitine plasma levels did not differ in the three Pugh-Child's classes; however, a trend towards higher levels of acetylcarnitine was found in Pugh-Child's class C.

In conclusion, the high levels of acetylcarnitine, short chain acylcarnitine, total esterified carnitine and total carnitine found in cirrhosis were linked to liver disease. Alcohol abuse seemed only to be an exacerbating factor.

\section{Introduction}

$L$-Carnitine (L-(-)-hydroxy-4-trimethylaminobutyrate) is an ubiquitous molecule in animal tissues involved primarily in the $\beta$-oxidation of long chain fatty acids (1). A carrier process involving carnitine is essential for long chain fatty acid transport across the inner mitochondrial membrane so that they may reach the mitochondrial matrix where $\beta$-oxidation occurs (2).

However, carnitine is also implicated in liver ketogenesis (3), in buffering the intracellular acyl-CoA/CoA ratio (4), in the oxidation of branched chain amino acids (5) and in peroxisomal $\beta$-oxidation (6).
In humans the last enzyme in carnitine synthesis, $\gamma$ butyrobetaine hydroxylase, is present only in liver and kidney and, in small amounts, in brain (7). Therefore, carnitine for the metabolic needs of all other tissues is provided by synthesis in the liver and kidney, and by the alimentary intake. Plasma carnitine constitutes the pool supplying all the tissues where carnitine synthesis cannot be carried out. In plasma, carnitine is present as free carnitine (molecule without an attached acyl group) and esterified carnitine. Esterified carnitine comprises a group of carnitine acylesters with carbon-chain of different length. According to their acid solubility, acylcarnitines are subdi- 
vided into short-chain acylcarnitine, in which the acyl moiety contains less than 10 carbon atoms, and longchain acylcarnitine, in which the acyl moiety contains more than 10 carbon atoms. The ratios between the fractions of carnitine in plasma is considered to express the corresponding ratios in hepatic tissue (2) which, in turn, reflects the acylation of coenzyme A in liver tissue (8).

In liver cirrhosis, both reduced liver synthesis or malnutrition could account for the low carnitine plasma levels found by Rudman et al. (9). However, more recently Fuller et al. (10) and Cooper et al. (11) did not find hypocarnitinaemia in alcoholic liver disease. They actually found high levels of total carnitine, due to an increased amount of esterified carnitine in chronic alcoholic liver disease. However, it is not clear if this finding is linked to alcohol, or to liver disease per se. Moreover, some authors (12) have reported a relationship between the severity of liver disease and the level of esterified carnitine, whereas others (10, 13) have not found this.

To clarify whether the alterations of carnitine plasma levels in liver cirrhosis are linked to alcohol consumption, to the severity of liver damage, or to the nutritional status of patients, a transversal study was carried out on inpatients suffering from liver cirrhosis of different aetiology and severity.

In cirrhotics, significantly increased levels of short chain acylcarnitine and a trend towards increased acetylcarnitine accounted for higher levels of esterified and total carnitine which did not depend primarily on alcohol abuse, or on nutritional status. Liver disease per se seemed to be implicated in altered carnitine plasma levels.

\section{Materials and Methods}

We studied 41 patients (27 males and 14 females) aged $55 \pm 11$ years (mean \pm S. D.) suffering from liver cirrhosis, and 16 healthy controls ( 11 males and 5 females) aged $43 \pm 13$ years. Twenty three patients had alcoholic cirrhosis, $4 \mathrm{HBsA} g$ positive cirrhosis, 13 cryptogenic cirrhosis and 1 biliary cirrhosis. Diagnosis was by case history and clinical findings, and confirmed by liver biopsy in 37 cases; 17 had ascites. No one suffered from gastrointestinal bleeding in the month prior of the study.
Seven cirrhotics were in Pugh-Child's class A, 20 in class B and 14 in class C. All were discharged alive from the hospital.

Diets were variable. Those with ascites received a sodium restricted diet with $58 \mathrm{~g}$ of protein, $40 \mathrm{mg}(250 \mu \mathrm{mol})$ of carnitine, $2.7 \mathrm{~g}(18.8 \mathrm{mmol})$ of lysine and $0.85 \mathrm{~g}(5.7 \mathrm{mmol})$ of methionine daily, the others received a free hospital diet containing about $70 \mathrm{~g}$ of protein, $60 \mathrm{mg}(370 \mu \mathrm{mol})$ of carnitine, $3.2 \mathrm{~g}(21.9 \mathrm{mmol})$ of lysine and $1.10 \mathrm{~g}(7.4 \mathrm{mmol})$ of methionine daily.

The nutritional status was assessed by:

1) the ratio of 24 hours creatinine excretion to height (creatinine/height ratio),

2) the mid upper arm muscle circumference calculated according to the formula: mid upper arm muscle circumference $=$ arm circumference $-\pi \times$ skinfold,

3) the triceps skinfold measured with a caliper.

The nutritional indices and the main liver function indices (measured with a routine commercial kit from Boehringer, West-Germany) are summarized in table 1 . In all patients renal function was assessed by measuring creatinine clearance. In two of them it was severely reduced ( 8 and $18 \mathrm{ml} / \mathrm{min}$ ).

Blood samples drawn between $7 \mathrm{a} . \mathrm{m}$. and $8 \mathrm{a} . \mathrm{m}$. after an overnight fast were collected into heparinized tubes and the plasma separated by centrifugation. Plasma samples of all cirrhotics and controls were assayed for total carnitine, short chain acylcarnitine, and long chain acylcarnitine according to $D i$ Donato (14); acetylcarnitine was measured according to Cooper et al. (15) in 19 cirrhotics and in 3 controls. The reproducibility ( $\%$ coefficient of variation) $2-4 \%$.

\section{Statistics}

The comparison of carnitine plasma levels and esterification ratios in cirrhotics and controls was carried out by the MannWhitney's test on the ranked values, because they did not fit the Gaussian distribution in the goodness of fit test. Only long chain acylcarnitine and long chain acylcarnitine/free carnitine were found to be normally distributed, so they were compared by the Student's t test. Even though carnitine plasma levels are higher in men (16), we did not subdivide our series in relation to sex to compare controls and cirrhotics, because the females/ males ratio was comparable in the two groups.

The relationship of carnitine plasma levels and esterification ratios with the degree of hepatic disease, expressed by the PughChild's class, was evaluated by the Spearman's test. Spearman's rank correlation was also used to assess possible associations between carnitine plasma levels and creatinine clearance.

To ascertain the degree of association of nutritional and liver disease indices with plasma carnitine, step by step multivariate analysis was done, using "Microstat" software (Ecosoft, Inc. 1987).

Tab. 1. Summary of the main clinical and biochemical data in cirrhotic patients.

\begin{tabular}{lclc}
\hline Alanine aminotransferase & $71 \pm 57 \mathrm{U} / 1$ & Albumin & $34 \pm 7 \mathrm{~g} / \mathrm{l}$ \\
Aspartate aminotransferase & $83 \pm 61 \mathrm{U} / 1$ & Triceps skinfold & $13.3 \pm 6.6 \mathrm{~mm}$ \\
$\gamma$-Glutamyltransferase & $185 \pm 213 \mathrm{U} / 1$ & Creatinine/height ratio & $83 \pm 34 \%$ \\
Alkaline phosphatase & $160 \pm 97 \mathrm{U} / 1$ & Mid upper arm circumference & $24 \pm \pm 2 \mathrm{~cm}$ \\
Bilirubin & $29 \pm 50 \mathrm{mg} / \mathrm{l}$ & Creatinine & $1.0 \pm 1.0 \mathrm{mg} / \mathrm{dl}$ \\
Prothrombin time & $58 \pm 12 \%$ & &
\end{tabular}




\section{Results}

Cirrhotics had higher levels of total and esterified carnitine with more widespread distributions which were not Gaussian (fig. 1). Only long chain acylcarnitine was substantially unchanged in cirrhotics compared with controls.
The short chain acylcarnitine/free carnitine ratio was significantly higher in cirrhotics; they also showed a trend towards a higher acetylcarnitine/free carnitine ratio (tab. 2).
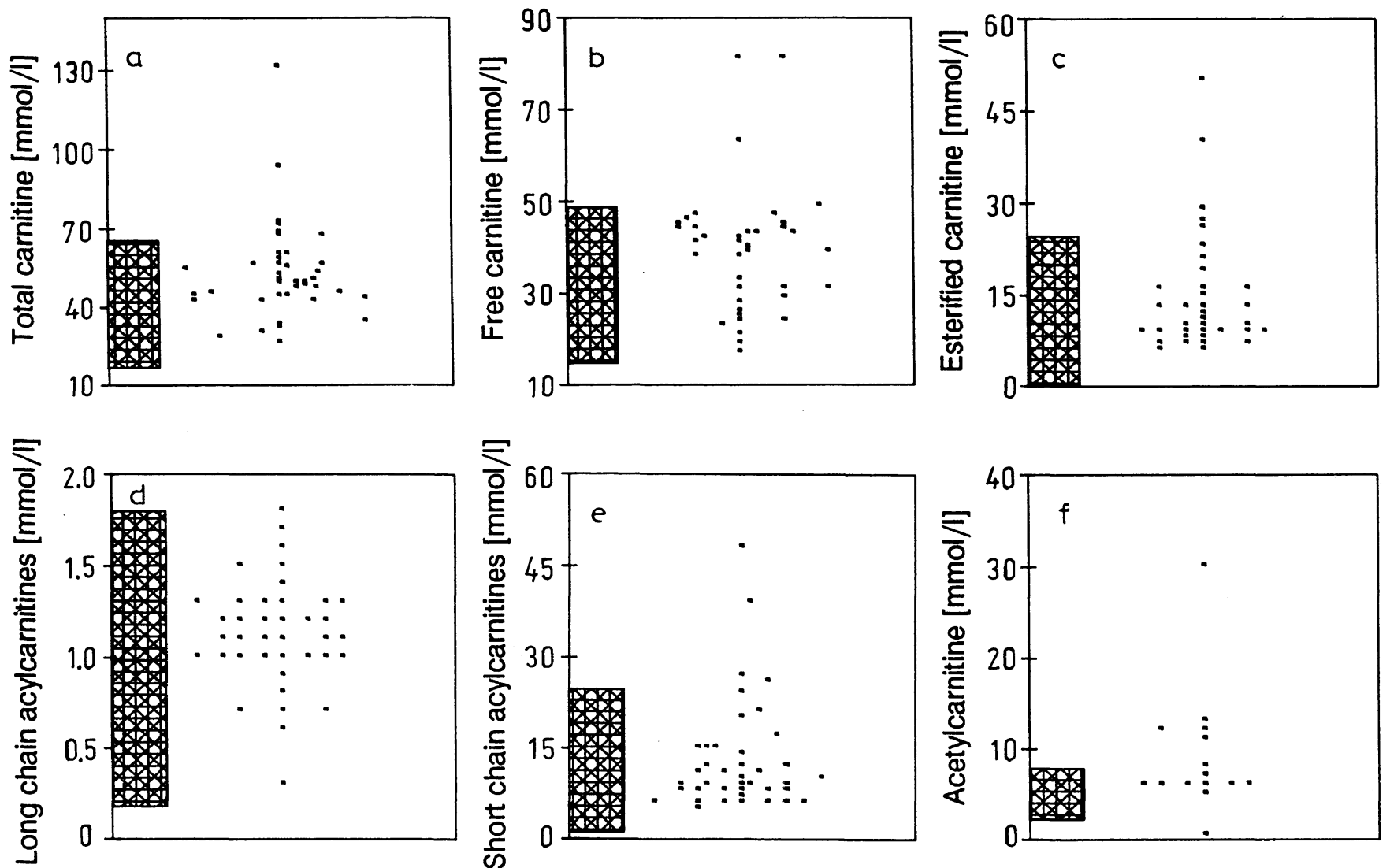

Fig. 1. Plasma levels of total carnitine and of its fractions in controls (shadowed) and in cirrhotics.

Each point represents one cirrhotic patient. Identical values of different patients are drawn horizontally.

\begin{tabular}{|c|c|c|c|c|c|c|}
\hline & $\mathrm{a}$ & $\mathrm{b}$ & $\mathrm{c}$ & $\mathrm{d}$ & $\mathrm{e}$ & $\mathrm{f}$ \\
\hline Cirrhotics* & $53 \pm 20$ & $39 \pm 14$ & $14 \pm 9$ & $1.1 \pm 0.3$ & $13 \pm 9$ & $8 \pm 5$ \\
\hline Controls* & $44 \pm 11$ & $36 \pm 7$ & $8 \pm 6$ & $1.0 \pm 0.3$ & $7 \pm 6$ & $5 \pm 1$ \\
\hline $\mathrm{U}$ & 211 & 301 & 171 & & 117 & 10 \\
\hline $\mathrm{t}$ & & & & 1.44 & & \\
\hline $\mathrm{p}$ & $<0.02$ & $<0.35$ & $<0.003$ & $<0.1$ & $<0.004$ & $<0.05$ \\
\hline
\end{tabular}

* values in $\mathrm{mmol} / \mathrm{l}$

Tab. 2. Mean values of the esterification ratios of carnitine in cirrhotics and controls.

\begin{tabular}{lllrr}
\hline & Cirrhotics & Controls & U & P \\
\hline Acetylcarnitine/free carnitine & $0.21 \pm 0.16$ & $0.14 \pm 0.03$ & 18 & 0.150 \\
Short-chain acylcarnitine/free carnitine & $0.36 \pm 0.26$ & $0.18 \pm 0.17$ & 159 & 0.001 \\
Long-chain acylcarnitine/free carnitine & $0.032 \pm 0.012$ & $0.027 \pm 0.007$ & $1.33^{*}$ & 0.098 \\
Total esterified carnitine/free carnitine & $0.39 \pm 0.26$ & $0.23 \pm 0.18$ & 186 & 0.006 \\
\hline
\end{tabular}

* t value 


\section{Carnitine and nutrition}

No relationship was found between nutritional indices and carnitine plasma levels or carnitine esterification ratios, with the exception of a weak direct correlation between triceps skinfold and long chain acylcarnitine (fig. 2).

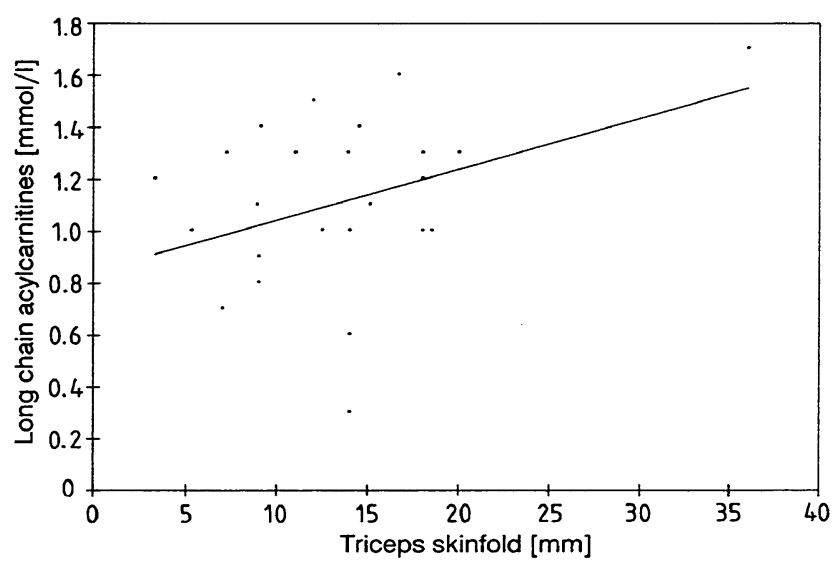

Fig. 2. Correlation between triceps skinfold and long-chain acylcarnitine.

$\mathrm{N}=23, \mathrm{r}=0.39, \mathrm{p}=0.052$

\section{Carnitine and liver function}

As far as the indices of liver disease are concerned, $\gamma$-glutamyltransferase showed the higher correlation with carnitine metabolism: $\gamma$-glutamyltransferase plasma activity was found to be directly correlated with acetylcarnitine (partial $\mathrm{r}^{2}=0.60, \mathrm{P}<0.01$ ), acylcarnitine/free carnitine (partial $\mathrm{r}^{2}=0.45, \mathrm{P}<0.01$ ), short chain acylcarnitine/free carnitine (partial $\left.\mathrm{r}^{2}=0.38, \mathrm{P}<0.05\right)$ and total esterified carnitine/free carnitine (partial $\mathrm{r}^{2}=0.38, \mathrm{P}<0.05$ ) (fig. 3). Also albumin showed a weak, inverse correlation with acetylcarnitine (partial $\mathrm{r}^{2}=0.036, \mathrm{P}=0.05$ ). Moreover, weak direct correlations between alkaline phosphatase and short chain acylcarnitine (partial $\mathrm{r}^{2}=0.12$, $\mathrm{P}<0.05$ ) and between alkaline phosphatase and total esterified carnitine (partial $\mathrm{r}^{2}=0.12, \mathrm{P}<0.05$ ) were found.

\section{Carnitine and the severity of cirrhosis}

Plasma carnitine levels and carnitine esterification did not differ significantly in the three Pugh-Child's classes. However a trend towards higher levels of acetylcarnitine in class $\mathrm{C}$ was evident (tab. 3).
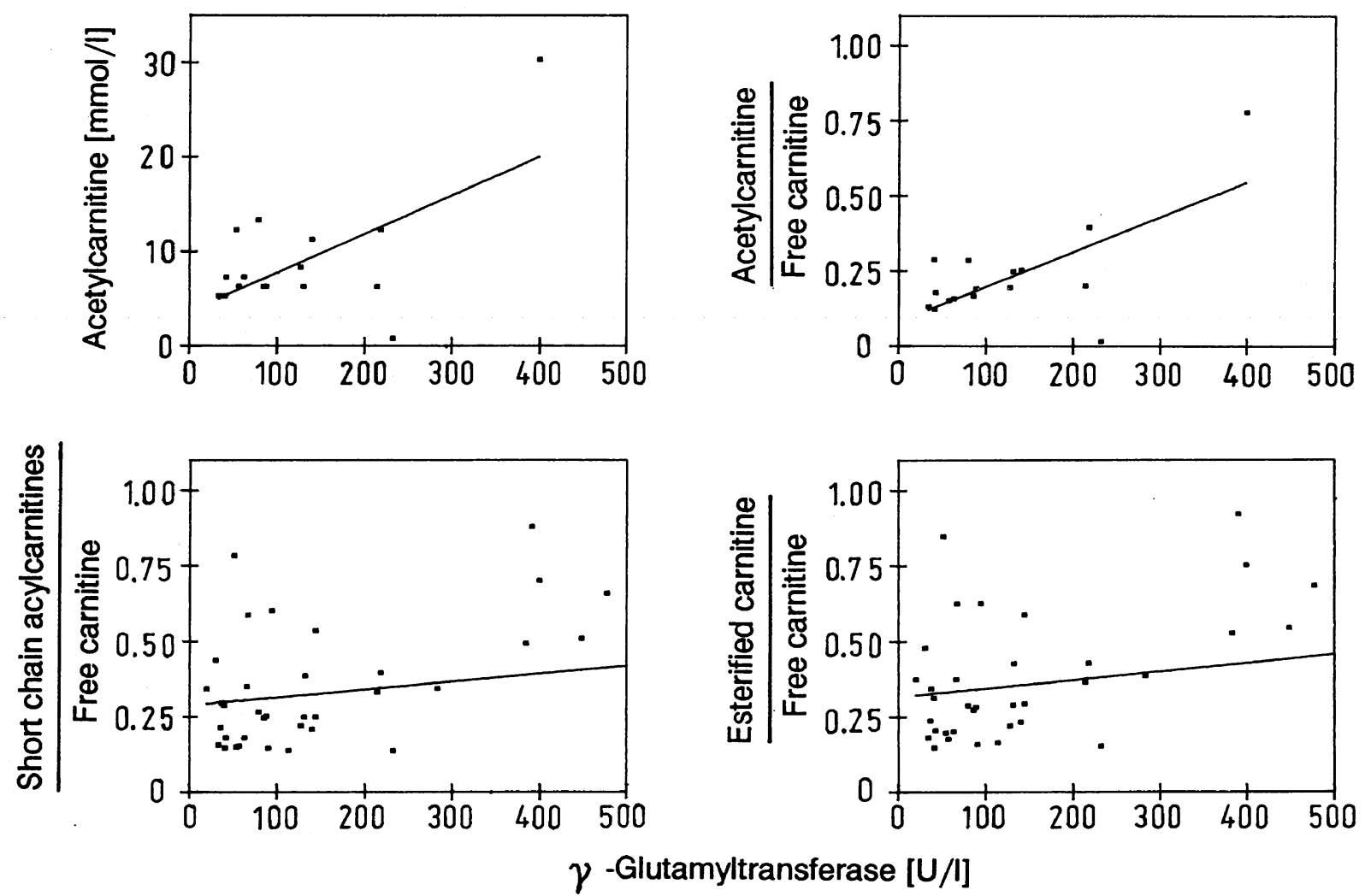

Fig. 3. Correlations of $\gamma$-glutamyltransferase with acetylcarnitine and the ratios of the acetylcarnitine/free carnitine ratios, short chain acylcarnitine/free carnitine ratios, and total esterified carnitine/free carnitine ratios. 
Tab. 3. Carnitine plasma levels and esterification ratios in the three classes of Pugh-Child.

\begin{tabular}{|c|c|c|c|c|c|c|c|}
\hline \multirow[b]{2}{*}{ Total carnitine } & \multirow[b]{2}{*}{$(\mathrm{mmol} / \mathrm{l})$} & \multicolumn{2}{|l|}{ A } & \multicolumn{2}{|l|}{ B } & \multicolumn{2}{|l|}{$\mathrm{C}$} \\
\hline & & 53 & +13 & 50 & +22 & 53 & +13 \\
\hline Free carnitine & $(\mathrm{mmol} / \mathrm{l})$ & 38 & \pm 11 & 36 & +14 & 39 & \pm 11 \\
\hline Total esterified carnitine & $(\mathrm{mmol} / \mathrm{l})$ & 15 & \pm 13 & 14 & \pm 10 & 14 & \pm 7 \\
\hline Acetylcarnitine & $(\mathrm{mmol} / \mathrm{l})$ & 6 & \pm 2 & 7 & \pm 2 & 12 & \pm 10 \\
\hline Short-chain acylcarnitine & $(\mathrm{mmol} / \mathrm{l})$ & 14 & \pm 13 & 13 & \pm 10 & 12 & \pm 7 \\
\hline Long-chain acylcarnitine & $(\mathrm{mmol} / \mathrm{l})$ & 1.1 & \pm 0.5 & 1.0 & \pm 0.3 & 1.2 & \pm 0.2 \\
\hline Total esterified carnitine/free carnitine & & 0.48 & \pm 0.49 & 0.39 & \pm 0.21 & 0.36 & \pm 0.21 \\
\hline Acetylcarnitine/free carnitine & & 0.13 & \pm 0.04 & $0.18=$ & \pm 0.04 & 0.30 & \pm 0.25 \\
\hline Short-chain acylcarnitine/free carnitine & & 0.45 & $\pm \quad 0.49$ & $0.36=$ & \pm 0.21 & 0.33 & \pm 0.20 \\
\hline Long-chain acylcarnitine/free carnitine & & 0.03 & \pm 0.02 & $0.03=$ & $\pm \quad 0.01$ & 0.03 & \pm 0.01 \\
\hline
\end{tabular}

Tab. 4. Carnitine plasma levels and esterification ratios in alcoholic and non-alcoholic cirrhosis

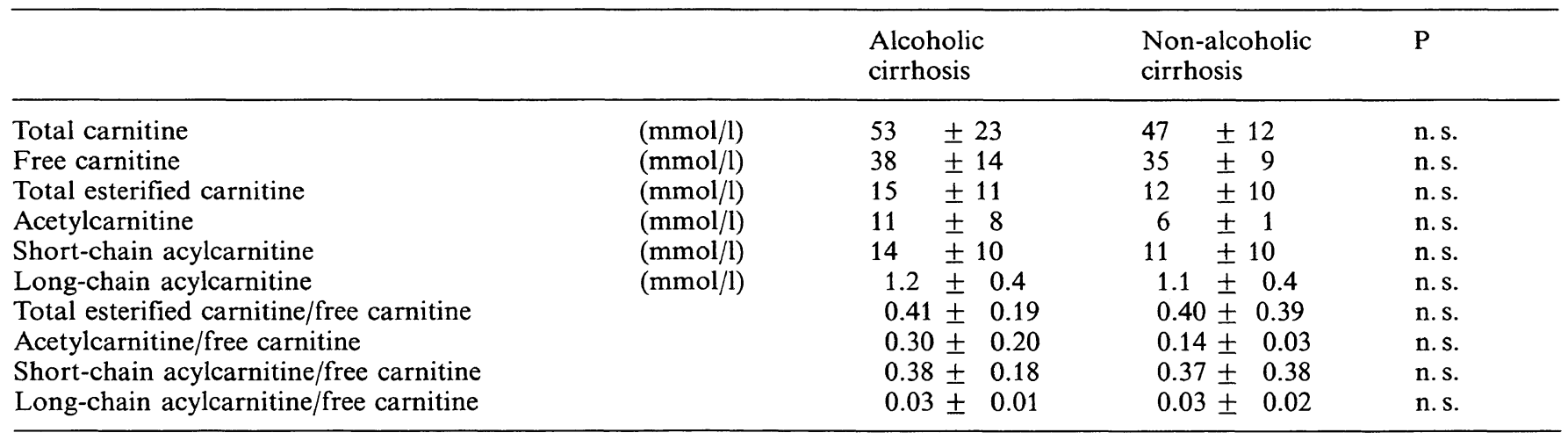

\section{Carnitine and alcohol intake}

Heavy drinkers with confirmed alcoholic HBsAg-negative cirrhosis did not differ significantly from nondrinkers with HBsAg-positive or cryptogenic cirrhosis with respect to carnitine, even though trends towards higher acetylcarnitine, and generally towards higher levels of carnitine esterification were found (tab. 4).

\section{Carnitine and renal function}

No relation was found between renal function and carnitine plasma levels or esterification ratios (tab. 5). The only two patients with severely reduced renal function (creatinine clearance $=8 \mathrm{ml} / \mathrm{min}$ and $18 \mathrm{ml} /$ min respectively) did not show alterations in carnitine plasma levels or esterification ratios.

Tab. 5. Values of the Spearman's rank correlation between carnitine plasma levels and creatinine clearance.

\begin{tabular}{lrl}
\hline & R & P \\
\hline Total carnitine & 0.038 & n.s. \\
Total esterified carnitine & -0.133 & n.s. \\
Short-chain acylcarnitine & -0.117 & n.s. \\
Total esterified carnitine/free carnitine & 0.193 & n.s. \\
Short-chain acylcarnitine/free carnitine & -0.173 & n.s. \\
Free carnitine & -0.181 & n.s. \\
Acetylcarnitine & 0.115 & n.s. \\
Long-chain acylcarnitine & -0.266 & n.s. \\
Acetylcarnitine/free carnitine & 0.014 & n.s. \\
Long-chain acylcarnitine/free carnitine & -0.344 & $<0.05$ \\
\hline
\end{tabular}

\section{Discussion}

Plasma levels of total carnitine, free carnitine and of short chain acylcarnitine in controls were in keeping with those of Cooper et al. (11) and of Fuller et al. (10), whereas long chain acylcarnitine was lower. This finding cannot be explained by differences of age and sex in our controls compared with the other two groups.

In fact, our controls should have had higher levels of carnitine, because of a greater prevalence of males, as carnitine has been reported to be higher in men than in women (16).

Moreover, differences in the method of measuring carnitine should be ruled out, as long chain acylcarnitine was also higher in Cooper's controls, which were assayed for carnitine by the same method that we used. It can be hypothesized that this finding is due to differences in the alimentary habitus in the studied groups, which were on a free diet and therefore conditioned by the alimentary custom of their own country.

As far as acetylcarnitine in controls is concerned, the subjects in which it was measured showed values in accordance with those reported by Cooper et al. (15).

The finding that cirrhotics showed a greater range of values of total carnitine, total esterified carnitine, and short chain acylcarnitine, with hypercarnitinaemia 
due to the increased amount of carnitine esterified with small acyl moieties, was in keeping with recent studies on carnitine in liver cirrhosis $(10-12)$. Thus, we were also unable to confirm Rudman's observations concerning hypocarnitinaemia in cirrhosis. However, it is plausible that Rudman studied more severely ill patients, as deduced from the high mortality rate in his series. In contrast, although $34 \%$ of our patients were in Pugh-Child's class C, all were discharged alive from the hospital.

\section{Carnitine and nutrition}

As far as nutritional indices and carnitine plasma level were concerned, the weak direct correlation between triceps skinfold and long chain acylcarnitine that we found is in keeping with the observation that in cirrhotics the amount of free fatty acids coming to the liver is linked to the amount of subcutaneous fat, expressed by triceps skinfold (17). In fact, in cells, free fatty acids must be activated to acyl-CoA to undergo $\beta$-oxidation. However, the acyl-CoA/CoA ratio is in equilibrium with the acylcarnitine/free carnitine ratio $(4,19)$, so that long chain acylcarnitine may reflect the amount of intracellular long-chain acyl-CoA, which depends on free fatty acids and on the rate of $\beta$-oxidation. In this regard, a direct correlation between triceps skinfold, an index of fatty store, and long chain acylcarnitine is to be expected.

We did not find the inverse correlation between creatinine/height ratio and short chain acylcarnitine observed by Fuller et al. (10). However, it is difficult to give a pathophysiological meaning to this correlation, which possibly signifies that the severer the cirrhosis, the higher is the level of short chain acylcarnitine and the lower the muscular mass. This hypothesis is deducible from the observations of D'Arienzo et al. (12), who found higher levels of esterified carnitine in class $\mathrm{C}$ cirrhotics and a direct correlation between esterified carnitine and glucagon, possibly implicated in the peripheral release of free fatty acids in cirrhotics (19).

In our series, however, the relationship between plasma carnitine and liver damage was not so clearcut. There was no link between the severity of liver disease evaluated by the Pugh-Child's classes and carnitine plasma levels or esterification ratios, although there was a trend towards a direct association of acetylcarnitine and acetylcarnitine/free carnitine with the severity of disease.

\section{Carnitine, liver function and alcohol intake}

As far as the single indices of liver disease were concerned, the direct correlations of $\gamma$-glutamyltransfer- ase with acetylcarnitine, acetylcarnitine/free carnitine and short chain acylcarnitine/free carnitine suggest that ethanol is involved in the increased esterification of carnitine with small acyl moieties in cirrhosis, because intra-hepatocytarian and plasma levels of $\gamma$ glutamyltransferase are massively increased by ethanol (20). Moreover, ethanol causes a reduction in the redox potential of the hepatocytes (21) which, together with direct mitochondrial lesions (22), impairs $\beta$-oxidation (23) and depresses the citric acid cycle (24) where acetate is utilized. For these reasons an increased amount of incompletely oxidized fatty acids may be expected. This acyl "pressure" on CoA should increase short chain acylcarnitine/free carnitine and acetylcarnitine/free carnitine ratios.

However, the comparison of heavy drinking with nondrinking cirrhotics showed no significant differences in carnitine, even though a clear trend towards higher acetylcarnitine was evident in drinkers.

In our opinion, this means that cirrhosis per se is characterized by the increase of the short chain acylcarnitine/free carnitine ratio and, possibly the acetylcarnitine/free carnitine ratio, even though alcohol might be a co-factor in the pathophysiology of such a finding.

\section{Carnitine and the severity of cirrhosis}

It does not seem conceivable that liver function directly accounts for alterations in carnitine plasma levels or in carnitine esterification, because these did not differ in the three Pugh-Child's classes, with the exception of a slightly higher acetylcarnitine/carnitine ratio in class $\mathrm{C}$. In fact, the Pugh-Child's classification can be considered a reliable index of liver function, because it is strictly correlated both with the aminopyrine breath test (26) and with the indocyanine green intrinsic hepatic clearance (27), two of the most valuable quantitative liver function tests.

The inverse correlation between albumin and acetylcarnitine was in keeping with the trend towards higher levels of acetylcarnitine in Pugh-Child's class C, but it was so weak that something other than liver function seems to be involved. It may be hypothesized that an increased utilization of fatty acids as a fuel in cirrhotics (25), together with possible alterations in citric acid cycle are implicated in the pathophysiology of the high levels of short chain acylcarnitine and acetylcarnitine in liver cirrhosis.

Moreover, a possible link between the increase in short chain acylcarnitine and cholestasis cannot be ruled out; this is suggested by the direct, albeit weak, correlation with alkaline phosphatase and by the fact 
that alkaline phosphatase and $\gamma$-glutamyltransferase showed a relationship with the esterification of carnitine. On the other hand, it is well known that cholestasis affects lipid metabolism, causing various abnormalities in the plasma lipid pattern.

\section{Carnitine and renal function}

Carnitine is cleared primarily by the kidneys (28), so that it may be thought that the subtle reduction of renal function frequently found in liver cirrhosis (29) could be involved in the alterations of plasma carnitine. However, the lack of any relationship between creatinine clearance and plasma carnitine levels or esterification, together with the observation that the two patients with a severely impaired renal function had normal carnitine levels, ruled out this hypothesis in our series.

\section{References}

1. Fritz, J. B. (1963) Carnitine and its role in fatty acid metabolism. Adv. Lipid. Res. 1, 285-334.

2. Bremer, J. (1983) Carnitine - metabolism and functions. Physiol. Rev. 63, 1420-1479.

3. Mc Garry, J. D., Robles-Valdes, C. \& Foster, D. W. (1975) Role of carnitine in hepatic ketogenesis. Proc. Natl. Acad. Sci. USA 72, 4385-4388.

4. Bieber, L. L., Emaus, R., Valkner, K. J. \& Glatz, J. P. C. (1982) Possible functions of short-chain carnitine acyltransferase. Fed. Proc. 41, 2858-2862.

5. Paul, H. S. \& Adibi, S. A. (1978) Effect of carnitine on branch-chain amino acid oxidation by liver and skeletal muscle. Am. J. Physiol. 234, E494-499.

6. Bieber, L. L., Krahaling, J. B., Clarke, P. R. H., Valkner, K. J. \& Tolbert, N. E. (1981). Carnitine acyltransferases in rat liver peroxisomes. Arch. Biochem. Biophys. 211, 599604.

7. Rebouche, C. J. \& Enger, A. G. (1980) Tissue distribution of carnitine biosynthetic enzymes in man. Biochim. Biophys. Acta 630, 22-29.

8. Brass, E. P. \& Hoppel, C. L. (1980) Relationship between acid-soluble carnitine and coenzyme $\mathrm{A}$ pools in vivo. Biochem. J. 190, 495-504.

9. Rudman, D., Swell, C. W. \& Ansley, J. D. (1977) Deficiency of carnitine in cachectic cirrhotic patients. J. Clin. Invest. $60,716-723$

10. Fuller, D. K. \& Hoppel, C. L. (1983) Elevated plasma carnitine in hepatic cirrhosis. Hepatology 3, 554-558.

11. Cooper, M: B., Forte, C. A., Round, J. M., Johonson, R. D., Hughes, R. D., William, R. \& Jones, D. A. (1987) Plasma carnitine and alcohol induced liver damage. Med. Sci. Res. 15, 671-673.

12. D'Arienzo, A., Mattera, D., Ambrogio, G., Duranti, L., Zeuli, L. \& Mazzacca, G. (1985) Hypercarnitinemia in cirrhosis. Hepatology 5, 343.

13. Fuller, R. \& Hoppel, C. L. (1985) Reply to above cited letter by D'Arienzo et al. Hepatology $5,343$.

14. Di Donato, S., Cornelio, F., Storchi, G. \& Rimoldi, M. (1979) Hepatic ketogenesis and muscle carnitine deficiency. Neurology 29, 780-785.

\section{Conclusions}

In conclusion, a selective increase in short chain acylcarnitine, the short chain acylcarnitine/free carnitine ratio (and possibly of acetylcarnitine and the acetylcarnitine/free carnitine ratio), resulting in high levels of total esterified carnitine and total carnitine was found in liver cirrhosis irrespective of its aetiology. The nutritional status did not seem to be involved in such alterations, which are conceivably dependent on liver cirrhosis per se.

More research is necessary to clarify why these alterations occur and their clinical significance.In particular, it is necessary to determine whether they are only a signal of a particular kind of energetic metabolism in chronic liver disease, or whether they express a relative lack of free carnitine and free CoA, potentially harmful to liver cells.

15. Cooper, M. B., Forte, C. A. \& Jones, D. A. (1986) Citrate interference with the determination of acetylcarnitin: a method for its elimination. Clin. Chim. Acta 159, $291-$ 299.

16. Rossle, C., Kohse, K. D., Franz, H. C. \& Furst, P. (1985) An improved method for the determination of free and esterified carnitine. Clin. Chim. Acta 49, 263-268.

17. Riggio, O., Merli, M., Cantafora, A., Di Biase, A., Lalloni, L., Leonetti, F., Miazzo, P., Rossi-Fanelli, F., Tamburrano, G. \& Capocaccia, L. (1984) Total and individual free fatty acids in liver cirrhosis. Metabolism 33, 646-651.

18. Borum, P. R. (1983) Carnitine. Am. Rev. Nutr. 3, $233-$ 259.

19. Marco, J. (1983) Glucagon in cirrhosis of the liver. In: Glucagon (Lefèbvre, P. J., ed.) Berlin: Springer Verlag, pp. 492-503.

20. Ishjj, H. (1986) Gamma-glutamyltransferase activity in liver of alcoholics and its histochemical localization. Alcohol. Clin. Exp. Res. 10, 81-85.

21. Jauhonen, P., Baraona, E., Mixakawa, H. \& Lieber, C. S. (1982) Mechanism for selective perivenular hepatotoxicity of ethanol. Alcoholism 6, 350-357.

22. Lane, B. P. \& Lieber, C. S. (1966) Ultrastructural alterations in human hepatocytes following ingestion of ethanol with adequate diets. Am. J. Pathol. 49, 593-603.

23. Hasumura, Y., Teschke, R. \& Lieber, C. S. (1976) Characteristics of acetaldehyde oxidation in rat liver mitochondria. J. Biol. Chem. 251, 4908-4913.

24. Lieber, C. S. (1977) Metabolic aspects of alcoholism. Baltimore: University Park Press.

25. Owen, O. E., Reichle, F. A., Mazzoli, M. A., Kreulen, T., Patel, M. S., Elfenbein, I. B., Golsorkhi, M., Chang, K. H. Y., Rao, N. S., Sue, H. S. \& Boden, G. (1981) Hepatic, gut, and renal substrate flux rates in patients with hepatic cirrhosis. J. Clin. Invest. 68, 240-252.

26. Villineuve, J. P., Infante-Rivard, C., Ampelas, M., PomierLayrargues, G., Huet, P. M. \& Marleau, D. (1986) Prognostic value of the aminopyrine breath test in cirrhotic patients. Hepatology 6, 928-931. 
27. Merkel, C., Bolognesi, M., Finucci, G. F., Angeli, P., Caregaro, L., Rondana, M. \& Gatta, A. (1989) Indocyanine green intrinsic hepatic clearance as a prognostic index of survival in patients with cirrhosis. J. Hepatol. 9, 16-22.

28. Maebashi, M., Kawamura, N., Sato, M., Yoshinga, K. \& Suzuki, M. (1976) Urinary excretion of carnitine in man. J. Lab. Clin. Med. 87, 760-766.
29. Gatta, A., Merkel, C., Grassetto, M., Milani, L., Zuin, R. \& Ruol, A. (1982) Enhanced renal sympathetic tone in liver cirrhosis: evaluation by intrarenal administration of dihydroergocristine. Nephron 30, 364-367.

Dott. Piero Amodio

Istituto di Medicina Clinica

Cattedra di Clinica Medica II

Policlinico Universitario

via Giustiniani 2

I-35100 Padova 